\title{
Modeling and Vibration Suppression of Flexible Spacecraft Using Higher-Order Sandwich Panel Theory
}

\author{
Milad Azimi \\ Department of Mechanical Engineering, Islamic Azad University East Tehran Branch, Tehran, Iran, 18661-13118

\begin{abstract}
Morteza Shahravi and Keramat Malekzadeh Fard
Department of Aerospace and Mechanical Engineering, Malek Ashtar University of Technology, Tehran, Iran, $15875-1774$
\end{abstract}

(Received 16 May 2014; accepted: 1 March 2017)

This paper presents a novel approach for the modeling and vibration suppression of a flexible spacecraft during a large angle attitude maneuver. A Higher-Order theory is used to model the elastic behavior of solar panel appendages with surface bounded piezoelectric (PZT) patches that capture the transverse shear deformation effects through the thickness of the smart sandwich panel. With the implementation of an appropriate time scales transformation technique and using Singular Perturbation Theory (SPT), the spacecraft dynamic behavior has been divided into double slow and fast subsystems. Modified Sliding Mode (MSM) and Strain Rate Feedback (SRF) control theory have been used for attitude and vibration control simultaneously by global stability proof of the overall system, while the controllers accomplished their missions in coupled rigid/flexible dynamic domain without parasitic parameter interactions. Numerical simulation assesses the benefits of the proposed approach.

\section{NOMENCLETURE}

$a$
$\mathbf{C}$
$d_{31}$
$D_{3}$
$e_{\theta}$
$E_{3}$
$E$
$g_{s}, g_{a}$
$G$

$h_{b}, h_{c}, h_{p}, h_{t}$

I

$J_{h}$

$k_{i}(i=1: 4)$

$\mathbf{K}$

$L_{b}, L_{P}$

M

$n$

$P$

q

$R$

$S_{1}$

$S_{\theta}^{H}$

$T^{j},{ }^{k} T_{P}^{j}$ system
Hub dimension

Structural damping matrix

Equivalent PZT coefficient

PZT material electric charge density displacement

Attitude error

PZT material electric field strength

Young's Modulus

PZT sensors and actuator amplifier gains, respectively

Shear modulus

Bottom, Core, PZT, Top layers thickness, respectively

Moment of inertia

Hub moment of inertia

Positive design parameters to stabilize the

\section{Stiffness matrix}

Length of the flexible pannel and PZT patches, respectively

Mass matrix

Number of elements

Boundary layer width of the sliding surface

General coordinates

Reduced in magnitude

PZT material strain

Sliding surface

Kinetic energy hub with attached main $j$-th pannel and $k$-th PZT pairs on $j$-th pannel, respectively

\begin{tabular}{|c|c|}
\hline$u(x, y)$ & $\begin{array}{l}\text { Displacement in the longitudinal } x \text { - } \\
\text { direction of the appendages }\end{array}$ \\
\hline$V$ & Potential strain energy \\
\hline$w(x, y)$ & Lateral deflection of the appendage \\
\hline$x_{k}$ & $\begin{array}{l}\text { Starting } x \text {-coordinate of the } k \text {-th PZT } \\
\text { patches }\end{array}$ \\
\hline${ }^{k} y^{j}$ & $\begin{array}{l}\text { The distance from strating point of PZT } \\
\text { with respect to neutral axis of core }\end{array}$ \\
\hline$\delta$ & Variation \\
\hline$\varepsilon$ & Singular perturbation parameter \\
\hline$\varepsilon_{3}^{T}$ & PZT material permitivity \\
\hline$\eta_{s}, \eta_{a}$ & Sensor and actuator voltages \\
\hline$\varpi_{P}$ & PZT width \\
\hline$\theta$ & Hub angle \\
\hline$\theta_{d}$ & Desired hub angle \\
\hline$\rho, \rho_{P}$ & Appendage and PZT density, respectively \\
\hline$\sigma_{1}$ & PZT material stress \\
\hline$\psi(x)$ & Finite element shape functions \\
\hline$\tau_{y x}$ & Shear stress \\
\hline$\left({ }^{*}\right)$ & Time differentiation \\
\hline
\end{tabular}

\section{INTRODUCTION}

Recently, control problems for flexible space structures have attracted significant attention because of the important demands for decreasing the consumption of energy and limitation of the mass. Today's modern spacecrafts consist of a rigid hub and flexible solar sandwich panel appendages. These sandwich panel constructions consist of metal or composite face sheets and metallic honeycombs that play an important role to increase the ratio of stiffness to weight in structure configuration. A flexible space structure is required to not only execute precise motion for the purpose of pointing, tracking, etc., but 
also to be stabilized and dampen the vibrations that are naturally excited along the motion as fast as possible at the terminal point. ${ }^{1}$ In order to accurately predict the vibrational behavior of such a structure, a more sophisticated model with less restrictive assumptions is needed. Smart sandwich structures with distributed sensors and actuators have the capability to actively respond to a changing environment while offering significant weight savings and additional passive controllability through ply tailoring. Piezoelectric sensing and actuation of composite laminates is the most promising concept due to the static and dynamic control capabilities. Among various types of sandwich structure modeling approaches such as classical theory with no shear deformation (CPT), first order shear deformation theory (FOSDT), Ordinary theories (OSPT), various Higher-Order approaches, the one that is suitable for sandwich structures with metallic honeycomb core is the Higher-Order Sandwich Panel Theory (HSAPT). ${ }^{2}$ It has long been recognized that higher order theories provide an effective solution tool for accurately and efficiently predicting the deformation behavior of sandwich structures subjected to bending loads. A review and assessment of various theories for modeling sandwich composites with application to sandwich beams can be found in some references. ${ }^{3,4}$ Many authors have investigated the higher-order theories to model the free vibration response of sandwich structures ${ }^{5,6}$ but no researches have reported on the analysis of the dynamical behavior of sandwich panels in attitude maneuver yet.

Many critical studies for the spacecraft attitude control problem by sliding mode control (SMC) approach have been done. ${ }^{7,8}$ A critical key point of controller design based on the $\mathrm{SMC}$ is that it uses nonlinear feedback control with discontinues behavior of sliding surface in the state space. However, because of the robust nature of this kind of controller due to the switching functions of the structure, it is an effective method to attenuate disturbances and uncertainties. It has also been applied in a wide range of maneuvering control of flexible spacecraft. The discontinuous nature of SMC designs is well known for exciting high frequency un-modeled dynamics, especially when applied to physical systems that are modeled imperfectly. To avoid these hazards, several techniques such as defining continuous switching functions, ${ }^{9}$ improve the system's performance. However, the elastic motion of the flexible spacecraft will be affected by the attitude maneuvering operation, which causes undesirable vibrations. For the flexible spacecraft maneuvering, the attitude regardless of the system flexibility or control on the flexible members, large amplitude transient, and steady state oscillations may occur.

Attitude maneuver of rigid spacecraft can be done without a lot of vibration problems after reaching its desired attitude. The task of vibration suppression of a flexible spacecraft by means of smart structures such as PZT sensor/actuator pairs has been studied by control system designers. ${ }^{10}$ Active control methods can be used to damp out undesirable vibrations of a flexible structure. ${ }^{11,12}$ Some of the various methods used for vibration control in systems are the Strain Rate Feedback (SRF), ${ }^{10,11,13}$ Positive Position Feedback (PPF), periodic output feedback control, sliding mode control, fast output sampling feedback control, wave suppression method, independent modal space control method, modified independent modal space control method, mode localization, and optimum control based on the minimization of power supplied to the beam.

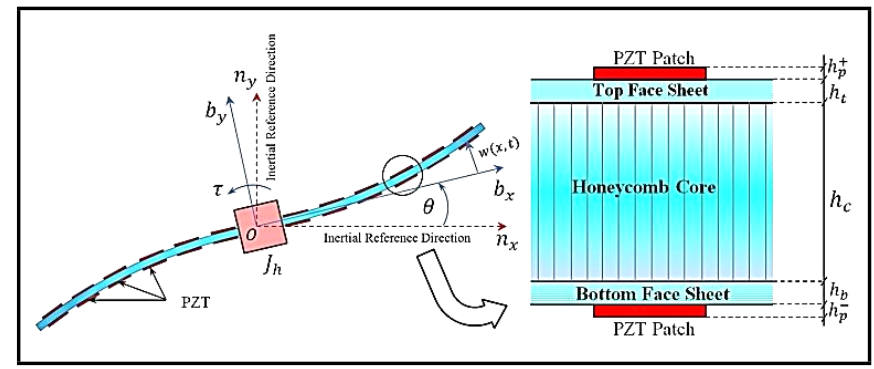

Figure 1. Flexible spacecraft model and parameters.

In this paper, a new hybrid control strategy is extended for flexible spacecraft rotational maneuver control as well as vibration suppression of the flexible sandwich appendages equipped by PZT patches by using singular perturbation theory. By introducing a novel approach by means of applying higher-order theories, the spacecraft structural domain is entered in its real configuration. To perform a desired maneuver while suppressing the vibration of the elastic modes, a timescale decomposition approach, where the coupled dynamics of the system are separated into fast (structural vibration) and slow (attitude dynamics) subsystems, is used. The derivation of the sliding mode control systems with switching functions imposes drastic chattering effects and excites low frequency modes of the systems. Hence, the modified SMC is adopted for the spacecraft attitude control to overcome this drawback. This modification of the attitude controller yields a shorter settling time and also decreases the residual vibration. In order to validate the performance and accuracy of the proposed approach, a comparative study is performed. Furthermore, numerical simulations are obtained to demonstrate the effectiveness and satisfaction of the proposed approach.

\section{MATHEMATICAL MODELING}

In the present study, we consider a system of spacecraft model which contain both rigid main body and the flexible sandwich panels, containing surface, bounded piezoelectric sensors, and actuators, as shown in Fig. 1. To identify the spacecraft attitude relative to an inertial frame $\mathbf{F}_{N}\left\{n_{x}, n_{y}, n_{z}\right\}$, a main body fixed frame $\mathbf{F}_{B}\left\{b_{x}, b_{y}, b_{z}\right\}$ is defined.

The velocity of a typical deformed point on each appendage with respect to the body of the reference frame is given by:

$$
\begin{aligned}
v^{i}(x, y, t)= & \left(\dot{u}^{i}(x, y, t)-w(x, y, t) \dot{\theta}(t)\right) \overrightarrow{b_{x}}+ \\
& \left(\dot{w}(x, y, t)+\left(x+a+u^{i}(x, y, t)\right) \dot{\theta}(t)\right) \overrightarrow{b_{y}}
\end{aligned}
$$

where $x$ and $y$ are the auxiliary variables measured from the root of the hub along and perpendicular to the un-deformed appendage axis. Henceforth, a similar system of superscript notation will be used in this paper. The deflections of the face sheets $u(x, y, t)$ and $w(x, y, t)$ are longitudinal and vertical displacement components measured along the body fixed frame, respectively. Moreover, the following assumptions are made: (1) Regarding to the ordinary form of higher-order sandwich panel $^{2}$ and related refined theories, ${ }^{14,15}$ a Quasi-layer wise hypothesis is considered in which the displacement fields of the upper and lower layers $u^{t, b}(x, y, t)$ and $w^{t, b}(x, y, t)$ satisfy the Euler-Bernoulli theory, and the core only undergoes 


$$
\begin{aligned}
& W\left\{w(x, y, t)=w(x, t)=w^{P+}(x, t)=w^{t}(x, t)=w^{c}(x, t)=w^{b}(x, t)=w^{P-}(x, t) ;\right.
\end{aligned}
$$

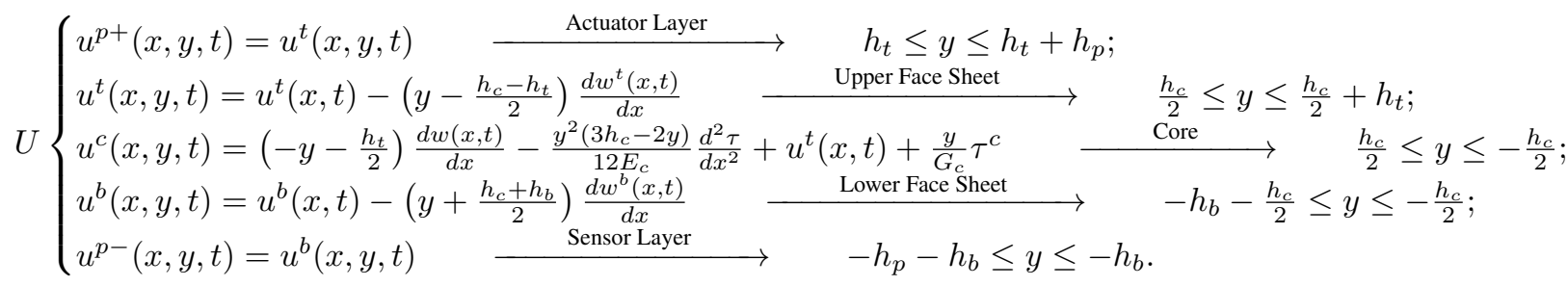

shear stresses $\tau_{y x}^{c} ;(2)$ the displacement field in the core was assumed to be linear in the vertical direction $w^{c}(x, y, t)$ and nonlinear in the longitudinal direction $u^{c}(x, y, t)$ in conformity with higher-order theory assumptions; (3) each layer has the same transverse deflection; ${ }^{16}$ (4) the mass and stiffness of the adhesive between the layers is neglected and; (5) loading only in the $b_{x}-b_{y}$ plane is considered, which solely caused deflections designed by $u$ and $w$.

The displacement of the smart panel including face sheets and the core, which consider Quasi Layer wise Theories (QLT) assumptions, are given by the Eq. (2).

Using Eq. (1), neglecting the radial deformations and nonlinear radial velocity, the total kinetic energy of the spacecraft model including PZT patches can be calculated by:

$$
\begin{aligned}
T= & \sum_{j=1}^{2} T^{j}+\sum_{j=1}^{2} \sum_{k=1}^{n_{k}}{ }^{k} T_{P}^{j} \\
= & \frac{1}{2} J_{h} \dot{\theta}^{2}+\frac{1}{2} \sum_{i}^{t, b, c} \int_{y_{S}^{-}}^{y_{S}^{+}} \int_{a}^{a+L_{b}} \rho^{j}\left(v^{i}\right)^{2} d x d y+ \\
& \frac{1}{2}\left[\int_{y_{S}^{+}}^{y_{P}^{+}} \int_{x_{k}}^{x_{k}+L_{P}} \rho_{P}^{j}\left(v^{t}\right)^{2} d x d y+\int_{y_{P}^{-}}^{y_{S}^{-}} \int_{x_{k}}^{x_{k}+L_{P}} \rho_{P}^{j}\left(v^{b}\right)^{2} d x d y\right]
\end{aligned}
$$

with

$$
\left\{\begin{array} { l } 
{ y _ { S } ^ { + } = h _ { c } / 2 + h _ { t } } \\
{ y _ { S } ^ { \overline { S } } = - h _ { b } - h _ { c } / 2 }
\end{array} \text { and } \left\{\begin{array}{l}
y_{P}^{+}=h_{c} / 2+h_{t}+h_{p} \\
y_{P}^{\bar{N}}=h_{p}-h_{b}-h_{c} / 2
\end{array} .\right.\right.
$$

According to the mentioned assumptions, the potential strain energy of the sandwich flexible structure, including sensor/actuator pairs, is considered to be

$$
\begin{aligned}
& V=\sum_{j=1}^{2} V^{j}+\sum_{j=1}^{2} \sum_{k=1}^{n_{k}}{ }^{k} V_{p}^{j} \\
&=\frac{1}{2} \sum_{i}^{t, b} \int_{a}^{a+L_{b}} E^{i}{ }^{j} I^{i}\left(\frac{\partial^{2} w(x, t)}{\partial x^{2}}\right)^{2}+{ }^{j} A^{i}{ }^{j} E^{i}\left(\frac{\partial u^{i}(x, t)}{\partial x}\right)^{2} d x+ \\
& \frac{1}{2} \int_{-\frac{h_{c}}{2}}^{\frac{h_{c}}{2}} \int_{a}^{a+L_{b}} G_{c} \gamma_{c}^{2} d x d y+\frac{1}{2} \sum_{i}^{t, b}{ }^{k} E_{P}^{j}\left({ }^{k} \varpi_{p}^{j}\right) h_{p} \\
& \int_{x_{k}}^{x_{k}+L_{P}} \Gamma^{j}\left(\frac{\partial^{2} w(x, t)}{\partial x^{2}}\right)^{2}+\left(\frac{\partial u^{i}(x, t)}{\partial x}\right)^{2} d x
\end{aligned}
$$

with

$$
\begin{aligned}
& \gamma^{c}=\frac{1}{h_{c}}\left(\left(u^{b}(x, t)-u^{t}(x, t)\right)-h^{c}+0.5\left(h_{t}+h_{b}\right)\right) \frac{d w(x, t)}{d x} \\
& { }^{k} \Gamma^{j}=\left({ }^{k} y^{j^{2}}+{ }^{k} y^{j k} h_{p}^{j}+\frac{{ }^{k} h_{p}^{j^{2}}}{3}\right) .
\end{aligned}
$$

The work done by the external torques $\tilde{\tau}$ and PZT patches ${ }^{17}$ is calculated by:

$$
\begin{aligned}
W_{n c} & =\sum_{j=1}^{2} W_{\tilde{\tau}}+\sum_{j=1}^{2} \sum_{k=1}^{n_{k}}{ }^{k} W_{p}^{j} \\
& =\frac{1}{2}\{\boldsymbol{\eta}\}^{T}[\Im]\{\boldsymbol{\eta}\}-\{\mathbf{q}\}^{T}[\mathbf{R}]\{\mathbf{q}\}+\tilde{\tau} \theta
\end{aligned}
$$

where

$$
\begin{aligned}
& {[\Im]=\operatorname{diag}\left({ }^{k} \xi_{p}^{j}\right) ; \quad[\mathbf{R}]=\left[\left\{{ }^{1} \Re_{p}^{j}\right\} \quad\left\{{ }^{2} \Re_{p}^{j}\right\} \ldots\left\{{ }^{n_{k}} \Re_{p}^{j}\right\}\right] ;}
\end{aligned}
$$

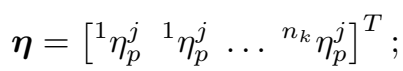

$$
\begin{aligned}
& { }^{k} \xi_{p}^{j}=\sum_{j=1}^{2} \sum_{k=1}^{n_{k}} \frac{{ }^{k} \varpi_{p}^{j} L_{P}}{{ }^{k} h_{p}^{j}}\left({ }^{k} \varepsilon_{3}^{j}{ }^{T}-{ }^{k} d_{31}^{j}{ }^{2} E_{P}^{j}\right) \\
& { }^{k} \eta_{p}^{j}=\sum_{j=1}^{2} k \sum_{k=1}^{n_{k}}{ }^{k} E_{3}^{j} \times{ }^{k} h_{p}^{j} \\
& { }^{k} \Re_{p}^{j}=\sum_{j=1}^{2} \sum_{k=1}^{n_{k}}{ }^{k} d_{31}^{j}{ }^{k} E_{P}^{j k} \varpi_{p}^{j}\left({ }^{k} y^{j}+\frac{{ }^{k} h_{p}^{j}}{2}\right) \int_{k^{j}}{ }^{x^{j}}\left\{\boldsymbol{\psi}^{\prime \prime}(t-b)(x)\right\}^{T} d x .
\end{aligned}
$$

Note that the work done by the $k$-th PZT patch is the combination of the conservative and non-conservative work terms, which are defined by integration over the volume of the PZT patches.

To generate an N DOF approximate differential equation model for a continuous system, the displacement $w(x, t)$ and $u^{t, b}(x, t)$ of the continuous system is expanded as a linear combination of $\mathrm{N}$ shape functions. The Hamilton's principle in its most general form is given by the variational statement:

$$
\int_{t_{1}}^{t_{2}}\left[\delta(T-V)+\delta W_{n c}\right] d t=0
$$

Integration over the spatial domains leads to the global mass, the stiffness, and the force matrices. In order to consider the structural damping effects in spacecraft dynamics, the 
Rayleigh's dissipation function may be given as:

$$
T_{d}=\frac{1}{2} \dot{\mathbf{q}}_{f}^{T} \mathbf{C} \dot{\mathbf{q}}_{f}
$$

Using Eqs. (3), (5), (7), (11), and the extended Hamilton's principle, the attitude dynamic model of the flexible spacecraft can be obtained in the following form:

$$
\begin{gathered}
{\left[\begin{array}{ll}
M_{R R} & \underline{\mathbf{M}_{R F}} \\
\mathbf{M}_{F R} & \underline{\underline{\mathbf{M}_{F F}}}
\end{array}\right]\left\{\begin{array}{c}
\ddot{\theta} \\
\ddot{\mathbf{q}}_{f}
\end{array}\right\}+\left[\begin{array}{cc}
0 & \underline{\mathbf{0}} \\
\underline{\mathbf{0}} & \underline{\underline{\mathbf{C}_{F F}}}
\end{array}\right]\left\{\begin{array}{c}
\dot{\theta} \\
\dot{\mathbf{q}}_{f}
\end{array}\right\}+} \\
{\left[\begin{array}{cc}
0 & \underline{\mathbf{0}} \\
\underline{\mathbf{0}} & \underline{\underline{\mathbf{K}_{F F}}}
\end{array}\right]\left\{\begin{array}{c}
\theta \\
\underline{\mathbf{q}} f_{f}
\end{array}\right\}=\left\{\begin{array}{c}
\tilde{\tau} \\
-[\mathbf{R}]\left[\mathbf{g}_{a}\right]\left\{\boldsymbol{\eta}_{a}\right\}
\end{array}\right\}} \\
\left\{\boldsymbol{\eta}_{S}\right\}=\left[\mathbf{g}_{s}\right][\Im]^{-1}[\mathbf{R}]^{T}\{\underline{\mathbf{q}\}}
\end{gathered}
$$

As it can be seen from Eq. (12), $\mathbf{R}$ and $\Im$ matrices can be decomposed into sensor and actuator parts corresponding to the sensor/actuator voltages $\boldsymbol{\eta}_{S}$ and $\boldsymbol{\eta}_{a}$. Controlling rigid-flexible systems is difficult because they are under-actuated systems in which all modes of flexure in each appendage have to be controlled by adjusting a single actuating torque. This essentially uses a perturbation parameter to divide the complex dynamic systems into simpler subsystems at different time scales. It has also been successfully applied to controlling systems with either flexible links or joints in combination with rigid bodies dynamic.

The crucial assumption to be made is that the spectrum of the flexible modes is well separated from the spectrum of the rigid modes. In this way, two reduced order systems are identified: a slow subsystem involves the hub variables as the slow state variables and the fast subsystem that contains the generalized flexible coordinates as the fast state variables. Then, a composite controller is designed to control these separated time-scale subsystems, such that when it is applied to the system with coupled rigid-flexible dynamics, the desired trajectory will be tracked with simultaneous control of appendages vibration. For this case, the dynamic model of the system is transformed into two-time-scale SPT model by defining a common scale factor $k_{\mathrm{Min}}$, which is the minimum of all the constants of the stiffness matrix $\mathbf{K}_{F F}$. With this common scale factor, the stiffness matrix $\underline{\overline{\mathbf{K}_{F F}}}$ elements with large magnitude in comparison to the other coefficients, in equation of the motion, can be scaled such that: ${ }^{18}$

$$
\begin{aligned}
& \left.\left[\underline{\underline{\mathbf{K}_{F F}}}\right]\left\{\mathbf{q}_{f}\right\}=k_{\text {Min }} \underline{\underline{\mathbf{K}_{F F}^{R}}}\right]\left\{\mathbf{q}_{f}\right\}=\{\mathbf{Z}\} \quad \Longrightarrow
\end{aligned}
$$

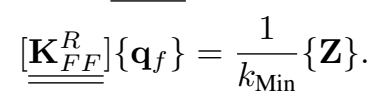

Introducing a new variable as a singular perturbation parameter $\varepsilon=\left(1 / k_{\mathrm{Min}}\right)^{0.5}$, Eq. (13) can be expressed as:

$$
\left.\underline{\underline{\left[\mathbf{K}_{F F}^{R}\right.}}\right]\left\{\mathbf{q}_{f}\right\}=\varepsilon^{2}\{\mathbf{Z}\}
$$

By comparing ${ }^{k} \Re_{p}^{j}$ matrix with $\sqrt{\varepsilon}$, it can be concluded that $O\left({ }^{k} \Re_{p}^{j}\right)=O(\varepsilon)$; therefore, ${ }^{k} \Re_{p}^{j}=\varepsilon^{k} \Re_{p}^{j}{ }^{R}$. Substituting Eq. (13) and Eq. (14) into the equations of motion, the system can be restated as follows

$$
\begin{aligned}
& \ddot{\theta}=\left[M_{R R}-\underline{\mathbf{M}_{R F}} \underline{\underline{\mathbf{M}_{F F}^{-1}}} \underline{\mathbf{M}_{F R}}\right]^{-1} \\
& \left(\tilde{\tau}-\underline{\underline{\mathbf{M}_{R F}}} \underline{\underline{\mathbf{M}_{F F}^{-1}}}\left\{-\varepsilon^{2} \underline{\underline{\mathbf{C}_{F F}}} \underline{\underline{\mathbf{K}_{F F}^{R-1}}} \dot{\mathbf{Z}}-\mathbf{Z}-\varepsilon[\mathbf{R}]\left[\mathbf{g}_{a}\right]\left\{\boldsymbol{\eta}_{a}\right\}\right\}\right) ; \\
& \left.\ddot{\mathbf{Z}}=\varepsilon^{-2} \underline{\underline{\underline{\mathbf{K}_{F F}^{R}}}} \underline{\underline{\mathbf{M}_{F F}^{-1}}}-\underline{\underline{\mathbf{M}_{F R}}} M_{R R}^{-1} \underline{\mathbf{M}_{R F}}\right]^{-1}- \\
& \left(\varepsilon[\mathbf{R}]\left[\mathbf{g}_{a}\right]\left\{\boldsymbol{\eta}_{a}\right\}-\varepsilon^{2} \underline{\underline{\mathbf{C}_{F F}}} \underline{\underline{\mathbf{K}_{F F}^{R-1}}} \dot{\mathbf{Z}}-\mathbf{Z}-\underline{\mathbf{M}_{F R}} M_{R R}^{-1} \tilde{\tau}\right)
\end{aligned}
$$

On setting in Eqs. (15) and (16), the slow subsystem becomes

$$
\mathbf{Z}_{\text {Slow }}=\left(\underline{\mathbf{M}_{F R}} M_{R R}^{-1} \tilde{\tau}-\varepsilon[\mathbf{R}]\left[\mathbf{g}_{a}\right]\left\{\boldsymbol{\eta}_{a}\right\}\right)
$$

Substituting Eq. (17) into Eq. (15), results in

$$
\begin{aligned}
\ddot{\theta}_{\text {Slow }}=\left(M_{R R}-\right. & \left.\underline{\mathbf{M}_{R F}} \underline{\underline{\mathbf{M}_{F F}^{-1}}} \underline{\underline{\mathbf{M}_{F R}}}\right)\left.^{-1}\right|_{\text {Slow }} . \\
& \left(1-\underline{\underline{\mathbf{M}_{R F}}} \underline{\underline{\mathbf{M}_{F F}^{-1}}} \underline{\left.\underline{\mathbf{M}_{F R}} M_{R R}^{-1}\right)\left.\tilde{\tau}\right|_{\text {Slow }} .}\right.
\end{aligned}
$$

To obtain the fast subsystem, a fast time scale defined by $\Phi=t / \varepsilon$ and setting $\mathbf{Z}_{\text {Fast }}=\mathbf{Z}-\mathbf{Z}_{\text {Slow }}$. Note that the slow variables remain constant in the fast time scale ${ }^{18}$ or due to the time scale introduced, the slow variables act as parameters. Also, by considering $\varepsilon^{2}=0$ in Eqs. (15) and (16), the fast subsystem can be calculated as

$$
\begin{aligned}
& \frac{d^{2}}{d \Phi^{2}} \mathbf{Z}_{\text {Fast }}=\underline{\underline{\mathbf{K}_{F F}^{R}}}\left[\underline{\underline{\mathbf{M}_{F F}}}-\underline{\underline{\mathbf{M}_{F R}}} M_{R R}^{-1} \underline{\underline{\mathbf{M}_{R F}}}\right]^{-1} \times \\
& \left\{\underline{\underline{\mathbf{M}_{F F}^{-1}}}\left(\left(-\underline{\underline{\mathbf{C}_{F F}}}\right) \frac{d}{d \Phi} \mathbf{Z}_{\text {Fast }}-\mathbf{Z}_{\text {Fast }}-\left.\left(\varepsilon[\mathbf{R}]\left[\mathbf{g}_{a}\right]\left\{\boldsymbol{\eta}_{a}\right\}\right)\right|_{\text {Fast }}\right)+\right. \\
& \left.\left.\underline{\mathbf{M}_{F R}^{-1} \tilde{\tau}}\right|_{\text {Fast }}\right\} \text {. }
\end{aligned}
$$

The equation of the motion, Eq. (12) is rewritten as

$$
\begin{aligned}
& {\left[\begin{array}{cc}
\mu_{R R} & \underline{\mathbf{0}} \\
\underline{\mathbf{0}} & \underline{\underline{\boldsymbol{\mu}_{F F}}}
\end{array}\right]\left\{\begin{array}{c}
\ddot{\theta}_{S} \\
\ddot{\mathbf{Z}}_{F}
\end{array}\right\}+\left[\begin{array}{cc}
0 & \underline{\mathbf{0}} \\
\underline{\mathbf{0}} & \underline{\underline{\mathbf{D}}}
\end{array}\right]\left\{\begin{array}{c}
\dot{\theta}_{S} \\
\dot{\mathbf{Z}}_{F}
\end{array}\right\}+} \\
& {\left[\begin{array}{cc}
0 & \underline{\mathbf{0}} \\
\underline{\mathbf{0}} & \underline{\underline{\boldsymbol{\kappa}_{F F}}}
\end{array}\right]\left\{\begin{array}{c}
\theta_{S} \\
\underline{\mathbf{Z}_{F}}
\end{array}\right\}=\left\{\begin{array}{c}
\left(1-\underline{\mathbf{M}_{R F}} \underline{\mathbf{M}_{F F}^{-1}} \underline{\mathbf{M}_{F R}} M_{R R}^{-1}\right) \tilde{\tau} \\
-\varepsilon[\mathbf{R}]\left[\mathbf{g}_{a}\right]\left\{\overline{\left.\boldsymbol{\eta}_{a}\right\}}-\underline{\mathbf{M}_{F R}} M_{R R}^{-1} \tilde{\tau}\right.
\end{array}\right\} .}
\end{aligned}
$$

where $\mu_{R R}, \boldsymbol{\mu}_{F F}, \mathbf{D}_{F F}$, and $\boldsymbol{\kappa}_{F F}$ are directly obtained from Eqs. (18) and (19). What can be deduced from the Eq. (20) is that the slow subsystem is not affected by PZT actuators. This feature of the system has enabled the torque devices, such as reaction wheels, to only stabilize the slow subsystem and prevent counteracting effects of two types of sub controllers.

\section{CONTROLLER DESIGN}

The ensuing tracking error is interpreted in SMC as a deviation of the system state from the nonlinear sliding surface $S$ and is defined as follows

$$
S_{\theta}^{H}=k_{3} e_{\theta}+k_{2} \dot{e}_{\theta}+k_{1} \dot{e}_{\theta}^{2} \tanh \left\{\dot{e}_{\theta} / p^{2}\right\} .
$$


The reduced order feedback control that would turn the sliding surface $S_{\theta}^{H}$ into an invariant manifold for the system is called the equivalent control $u_{\text {eq }}$ to ensure that $\dot{S}_{\theta}^{H}=0$. Additionally, the variable structure part $u_{\mathrm{VS}},{ }^{19}$ is selected in order to guarantee that $S_{\theta}^{H}=0$, thus the designing surface is attractive and the desired condition is accessible. The control law is written as

$$
u=u_{\mathrm{eq}}+u_{\mathrm{vs}} \text {. }
$$

The dynamics in sliding mode can be written as

$$
\dot{S}_{\theta}^{H}=0 \rightarrow \frac{d}{d t}\left(e_{\theta}\right)+\frac{d}{d t} f\left(\dot{e}_{\theta}\right)=0 .
$$

By solving the above equation for the control input, an expression for $u_{\mathrm{eq}}$ is obtained as follows

$$
u_{\mathrm{eq}}=-\frac{k_{3} \dot{e}_{\theta} A}{\aleph}
$$

where

$$
k_{2} \leq \aleph=\frac{d}{d \dot{e}_{\theta}} f\left(\dot{e}_{\theta}\right) ; \quad A=\left.\left(M_{R R}^{-1}-\underline{\mathbf{M}_{R F}^{-1}} \underline{\underline{\mathbf{M}_{F F}}} \underline{\underline{\mathbf{M}_{F R}^{-1}}}\right)\right|_{\text {Slow }} .
$$

Defining the variable structure component $u_{\mathrm{Vs}}$ yields

$$
u_{\mathrm{VS}}=\left.k_{4} S_{\theta}^{H}\right|_{\mathrm{P}-\mathrm{Amp}}
$$

where $k_{4}$ is a positive number and the term $\left.S_{\theta}^{H}\right|_{\mathrm{P}-\mathrm{Amp}}$ shows that the sliding surface's proportionate gain has been amplified by positive numbers $k_{3}$. This technique is employed to reduce the settling time in actuator which placed on the rigid main body.

Theorem: Let the control objective to force the rigid body modes to follow some pre-specified trajectories. Then, the control law Eq. (22) can achieve this objective and ensures that $S_{\theta}^{H}$ tends to zero as time tends to infinity.

Proof: Consider the following Lyapunov function candidate:

$$
\begin{aligned}
V(t)= & \left\{\begin{array}{ll}
\dot{e}_{\theta} & \underline{\dot{\mathbf{Z}}_{F}^{T}}
\end{array}\right\}[\boldsymbol{\mu}]\left\{\begin{array}{c}
\dot{e}_{\theta} \\
\dot{\mathbf{Z}}_{F}
\end{array}\right\}+ \\
& \left\{\begin{array}{ll}
e_{\theta} & \underline{\mathbf{Z}_{F}^{T}}
\end{array}\right\}\left[\operatorname{diag}\left(k_{4}, \underline{\underline{\boldsymbol{\kappa}_{F F}}}\right)\right]\left\{\begin{array}{c}
e_{\theta} \\
\underline{\mathbf{Z}_{F}}
\end{array}\right\} .
\end{aligned}
$$

The positive weighting coefficients $k_{i}$ are included to allow relative emphasis on the four contributors to the error energy of the system. This function (Eq. (27)) is one of many possible ways to weight the mechanical system error energy and merely provides one illustration of an approach. It is obvious by inspection that imposing $k_{i}>0$ in Eq. (27) guarantees $V(t) \geq 0$ and that the global minimum of $V(t)=0$ occurs only at the desired state.

The desired state implies that when the rigid hub begins at the rest and rotates to a new angular position $\theta=\theta_{d}=$ const, the time derivative to the Eq. (27) yields:

$$
\begin{aligned}
\dot{V}(t)= & 2\left\{\begin{array}{ll}
\dot{e}_{\theta} & \underline{\dot{\mathbf{Z}}_{F}^{T}}
\end{array}\right\}[\boldsymbol{\mu}]\left\{\begin{array}{c}
\ddot{e}_{\theta} \\
\ddot{\mathbf{Z}}_{F}
\end{array}\right\}+ \\
& 2\left\{\begin{array}{ll}
e_{\theta} & \underline{\mathbf{Z}_{F}^{T}}
\end{array}\right\}\left[\operatorname{diag}\left(k_{4}, \underline{\underline{\boldsymbol{\kappa}_{F F}}}\right)\right]\left\{\begin{array}{c}
\dot{e}_{\theta} \\
\dot{\mathbf{Z}}_{F}
\end{array}\right\} .
\end{aligned}
$$

The sliding condition should satisfy $\dot{S}_{\theta}^{H}=0$ and $S_{\theta}^{H}=$ 0 . In the case of $S_{\theta}^{H}=0$, where $e=-k_{2} \dot{e}_{\theta}-$ $k_{1} \dot{e}_{\theta}^{2} \tanh \left\{\dot{e}_{\theta} / p^{2}\right\} / k_{3}$, we have $\ddot{e}=-k_{3} \dot{e} / \aleph$, then Eq. (28) yields

$$
\dot{V}(t)=-2\left\{\begin{array}{ll}
\dot{e}_{\theta} & \underline{\dot{\mathbf{Z}}_{F}^{T}}
\end{array}\right\}[\hat{\boldsymbol{\mu}}]\left\{\begin{array}{c}
\dot{e}_{\theta} \\
\dot{\mathbf{Z}}_{F}
\end{array}\right\}
$$

where

$$
[\hat{\boldsymbol{\mu}}]=\left[\begin{array}{cc}
\frac{\mu_{R R} k_{3}}{\aleph}+\frac{k_{4} f\left(\dot{e}_{\theta}\right)}{\dot{e}_{\theta}} & \underline{\mathbf{0}} \\
\underline{\mathbf{0}} & \underline{\mathbf{D}_{F F}}
\end{array}\right] .
$$

Lemma: Let $S$ be the Schur complement of $\hat{\mu}(1,1)$ in $\hat{\boldsymbol{\mu}}$, that is

$$
S=\underline{\mathbf{D}_{F F}}-\hat{\mu}(1,2)\left(\frac{\mu_{R R} k_{3}}{\aleph}+\frac{k_{4} f\left(\dot{e}_{\theta}\right)}{\dot{e}_{\theta}}\right)^{-1} \hat{\mu}(2,1) .
$$

Then $[\hat{\boldsymbol{\mu}}]$ is positive definite if $\mu_{R R}(1,1)>0$ and $S>0$. Utilizing the SPT technique discretize the equation of the motion, thereafter the prescribed control law has implemented on $\theta$ coordinate which only needs $\mu_{R R}(1,1)>0$. Considering all possible values for $k_{i}$, it can be seen from Schur complement method that the Eq. (29) is a negative definite function of $\dot{e}_{\theta}$. It can be found that the only equilibrium point satisfies $\dot{V}(t) \cong 0$. So the designed sliding surface converges to origin asymptotically.

\section{SIMULATION RESULTS AND DISCUSSION}

In this section, a comparative numerical simulation is used to demonstrate the validity of the flexible spacecraft performance and the effectiveness of the proposed hybrid controller with the new substructure model approach (SPT technique). A uniform symmetrical sandwich panel with PZT layers which are bonded at the bottom and top surfaces is considered as flexible parts of a spacecraft. The spacecraft simulation involves a single-axis $60^{\circ}$ rest-to-rest maneuver. The parameters for the simulated flexible spacecraft are tabulated in Table 1. All computations presented in the paper are calculated by using MATLAB/Simulink software package. The results are summarized comparing the case with conventional flexible appendages, modeled by Euler-Bernoulli beams in the present study, and those available in open literature. In all the simulations, the initial conditions are considered as $x_{0}=\left[\begin{array}{ll}0 & \mathbf{0}\end{array}\right]^{T}$, $\theta_{d}=60^{\circ}, \dot{\theta}_{d}=\ddot{\theta}_{d}=0$.

Figure 2 shows the results of implementing the proposed hybrid controller in which the SMC using hyperbolic tangent function is utilized for the slow subsystem. Moreover, the imposed desired angular position is accurately achieved as well (Fig. 3). The controller gains $k_{1}, k_{2}, k_{3}$, and $k_{4}$ are selected to be $100,10,0.7$ and 1.2 , respectively.

As seen in Fig. 2, the convergence time for desired angular displacement of $60^{\circ}$ and velocity of $0^{\circ} / \mathrm{s}$ is about 40 seconds. The proposed controller, containing the proportionate amplification term is considered and the results of the simulations for the case that exclude the current term, are shown in these figures. As it can be seen, the settling time period in the case in which the P-Amp term is excluded, is much longer than the proposed controller.

Figure 3 shows the required equivalent control torque for performing the maneuver. For comparison, the conventional 


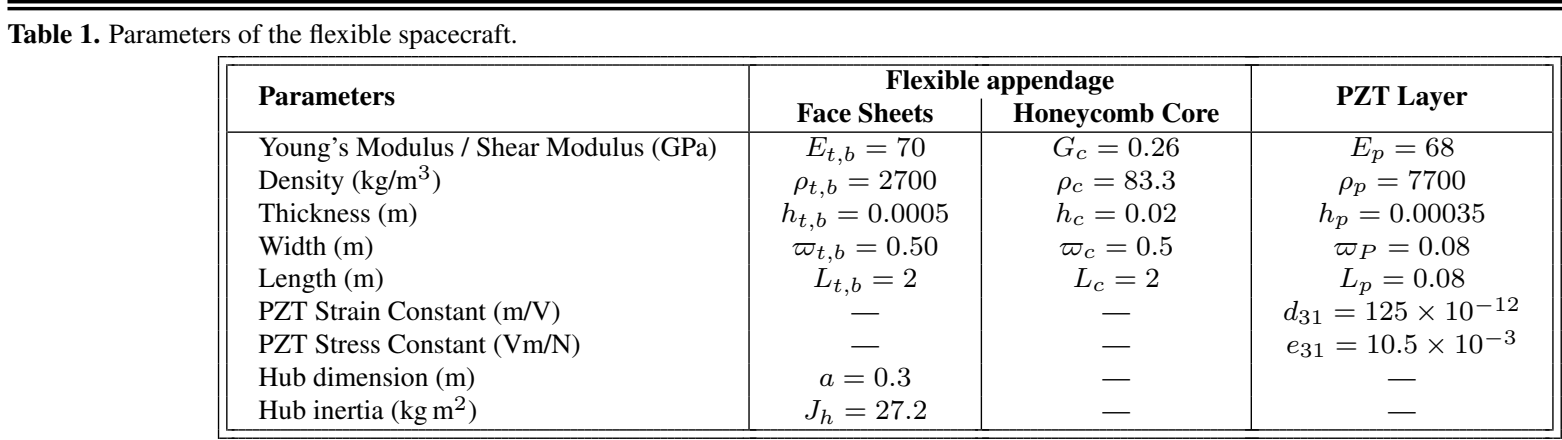



Figure 2. The attitude maneuver control integrated with SRF using the proposed and conventional controller; (a) The time history of attitude dynamics (slow subsystem) and (b) The time history of attitude velocity of the hub.

sliding mode control (CSMC), ${ }^{19}$ has been implemented. The CSMC that is designed in the time domain is hardly applicable as the inherent elastic modes of the flexible systems will be unduly excited by the switching control input. In order to overcome this drawback, the $\operatorname{sgn}($.$) term present in the con-$ ventional sliding surface design is replaced by a hyperbolic tangent function in order to reduce the effects of chattering.

Active control technique (SRF) and using PZT sensor/actuator patches in conjunction with an attitude controller (SMC) but in two separate sub controllers were used to reduce the residual vibration. Additionally, active suppression of structural vibration causes smooth and fine actuation of at-

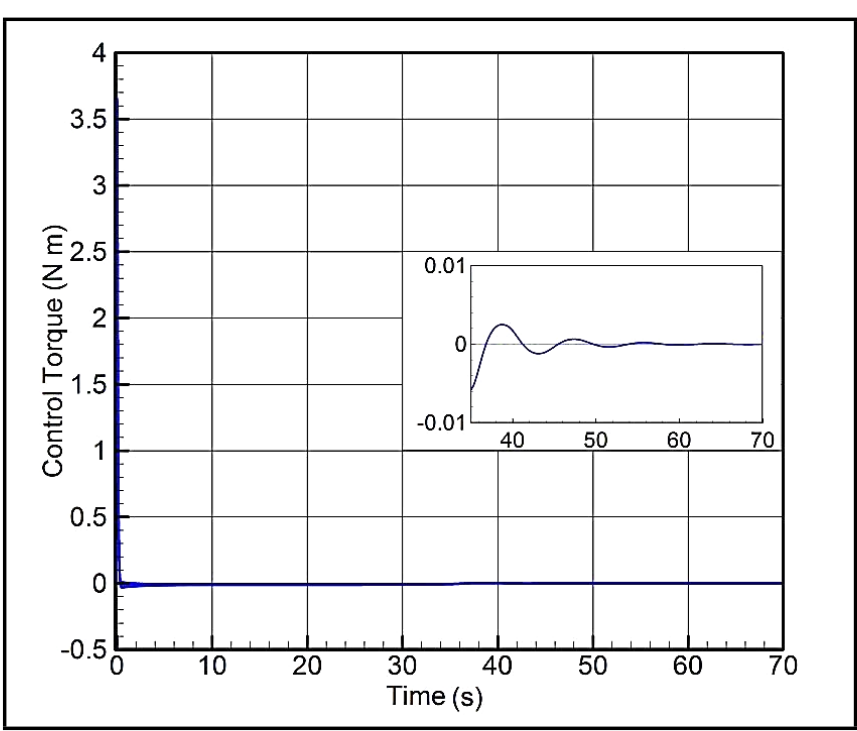

Figure 3. Time history of control actions applied on the slow subsystem.

titude controller. This is an important characteristic for actual implementation of the controller. Convergence of the flexible body coordinates and PZT actuation voltage are shown in Figs. 4 to 6 .

In the present study, actuators and sensors are placed as collocated sets of PZT at each appendage, and play the role of a MIMO control system. The potential capabilities of the PZT pairs are considered in two different instances: a condition in which the attitude controller and the PZT voltages are applied to the dynamic model and in order to compare the effects of PZT voltages on residual vibration suppression of the elastic appendages so an active control system is inactivated. These two cases are depicted in Figs. 5 and 6.

By comparing Fig. 4 with Fig. 5, it can be deduced that the effect of PZT actions is noteworthy. Notice that the main qualitative feature of the vibrational behavior analysis of the present study is based on the higher-order quasi-layer wise sandwich panel theory that is more easily perceived comparing with those formal with predefined configuration. Figures $4 \mathrm{a}$ and $4 \mathrm{~b}$ reveal that considering the shear stress and higher-order longitudinal deflection pattern for the core results to an inherent vibration profile. Furthermore, significant deviations are observed between the higher order theory and the classical panel theory in the vibrational behavior of the elastic appendages which indicates the importance of the higher order terms in the displacement field.

The primary conclusion drawn from Figs. 4 and 5 is that the HOSPM with a prescribed formulation yields accurate responses for three-layer sandwich substructures. Moreover, the assumption adopted by different researchers in literature is 


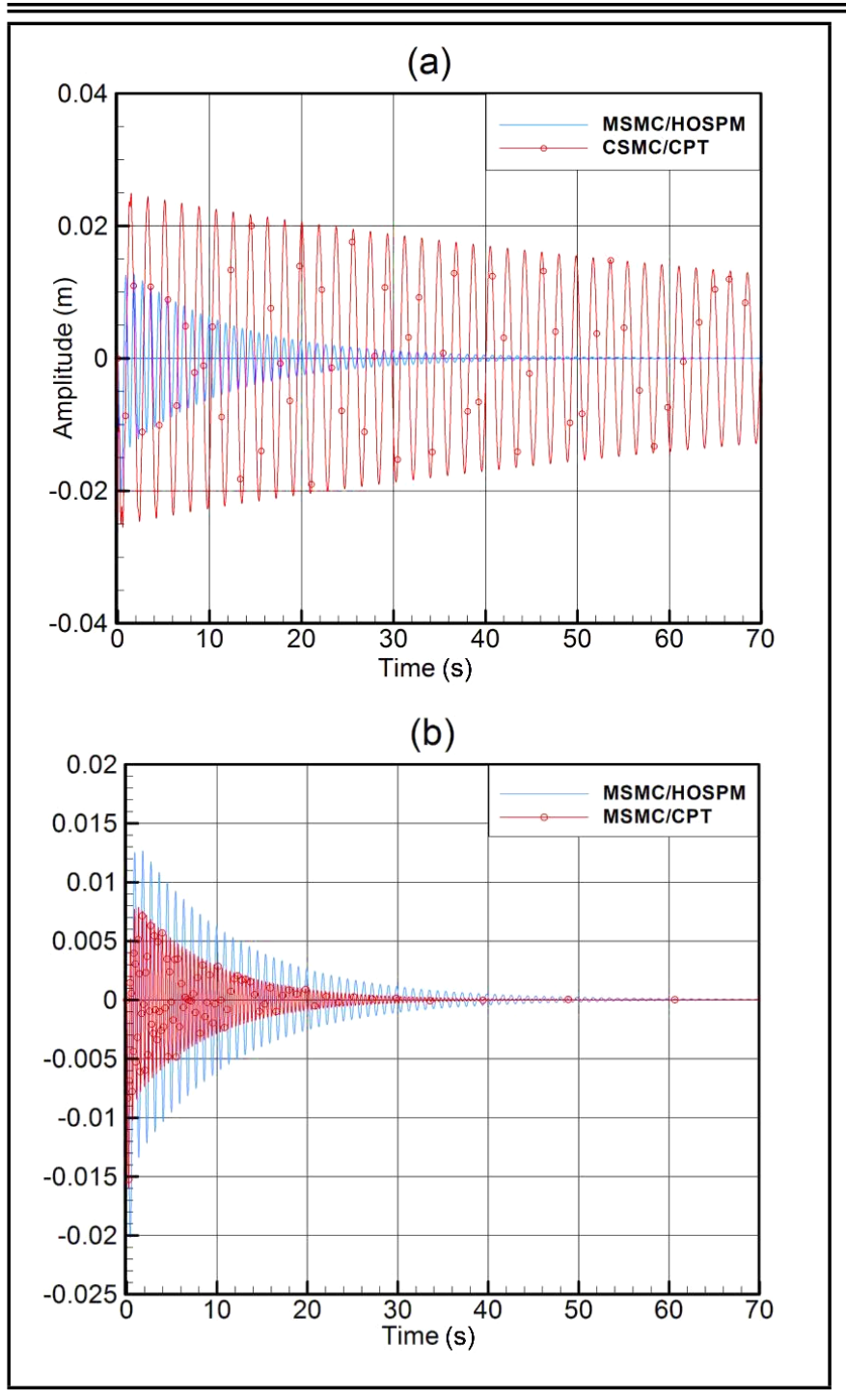

Figure 4. Time history of the appendage deflection without an active vibration control; (a) Modified SMC/Higher-Order SPM and SMC/CPT and (b) Modified SMC/Higher-Order SPM and Modified SMC/CPT.

based on that the dynamics of the space structures are exactly known and is examined and reported in Figs. 4a, 5a, and 6b. In the attitude control law, the modification to sliding manifold for slow subsystem ensures that the spacecraft follows the shortest possible path to the sliding manifold and highly reduce the switching action. As seen in Figs. 6a and 6b, this reduction causes the PZT actuation to be suppressed.

\section{CONCLUSIONS}

Higher-order sandwich panel theory has been used for dynamic modeling of smart flexible appendages of flexible spacecraft during attitude maneuver. The obtained equations decomposed into two different time scales and used singular perturbation theory. Based on the governing equations of motion, a hybrid control strategy has been developed for simultaneous attitude and vibration control during a large targeting maneuver. Using higher-order sandwich panel theory for modeling of elastic deflection of the flexible appendages improves the flexible/rigid body interaction effects of the spacecraft during attitude maneuver. It is found that the proposed model is useful to monitor extra vibrational behavior of the smart flexible appendages accurately from the view point of layer wise

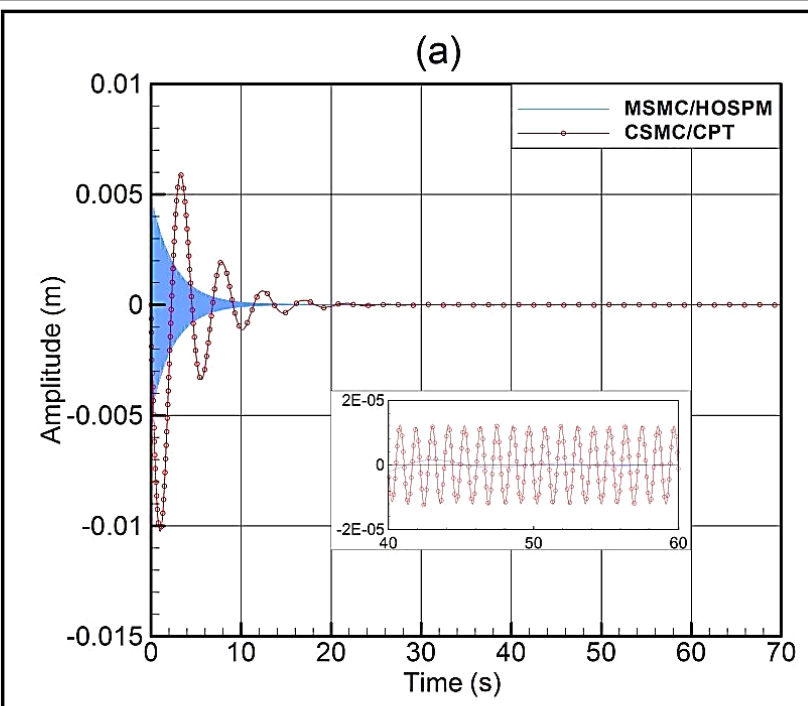

(b)

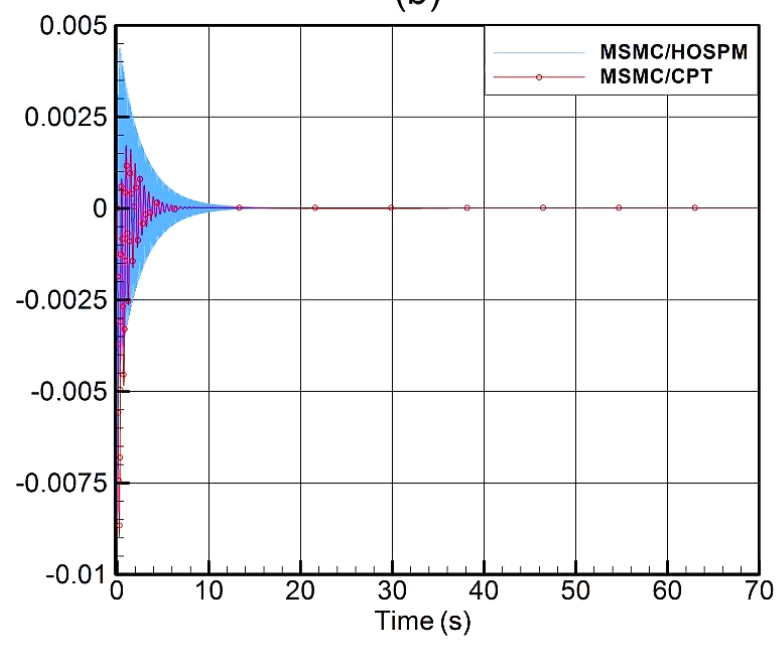

Figure 5. Time history of the appendage deflection with active vibration control; (a) Modified SMC/Higher-Order SPM and SMC/CPT and (b) Modified SMC/Higher-Order SPM and Modified SMC/CPT.

theory. Moreover, modifying the sliding surface reduces the issues of the excitation of high frequency elastic modes that frequently happen in the conventional sliding mode, whereas expedites the error convergence. Global stability of the coupled rigid/flexible system has been guaranteed via Lyapunov approach by implementation of singular perturbation theory during attitude and vibration control design procedure.

\section{REFERENCES}

1 Koch, R. M. Structural dynamics of large space structures having random parametric uncertainties, International Journal of Acoustics and Vibration, 8 (2), 95-103, (2003). https://dx.doi.org/10.20855/ijav.2003.8.2135

2 Frostig, Y. and Baruch, M. Free vibrations of sandwich beams with a transversely flexible core: a high order approach, Journal of Sound and Vibration, 176 (2), 195-208, (1994). https://dx.doi.org/10.1006/jsvi.1994.1368

$3 \mathrm{Hu}, \mathrm{H}$., Belouettar, S., Potier-Ferry, M, and Daya, E. M. Review and assessment of various theories for modeling sandwich composites, 


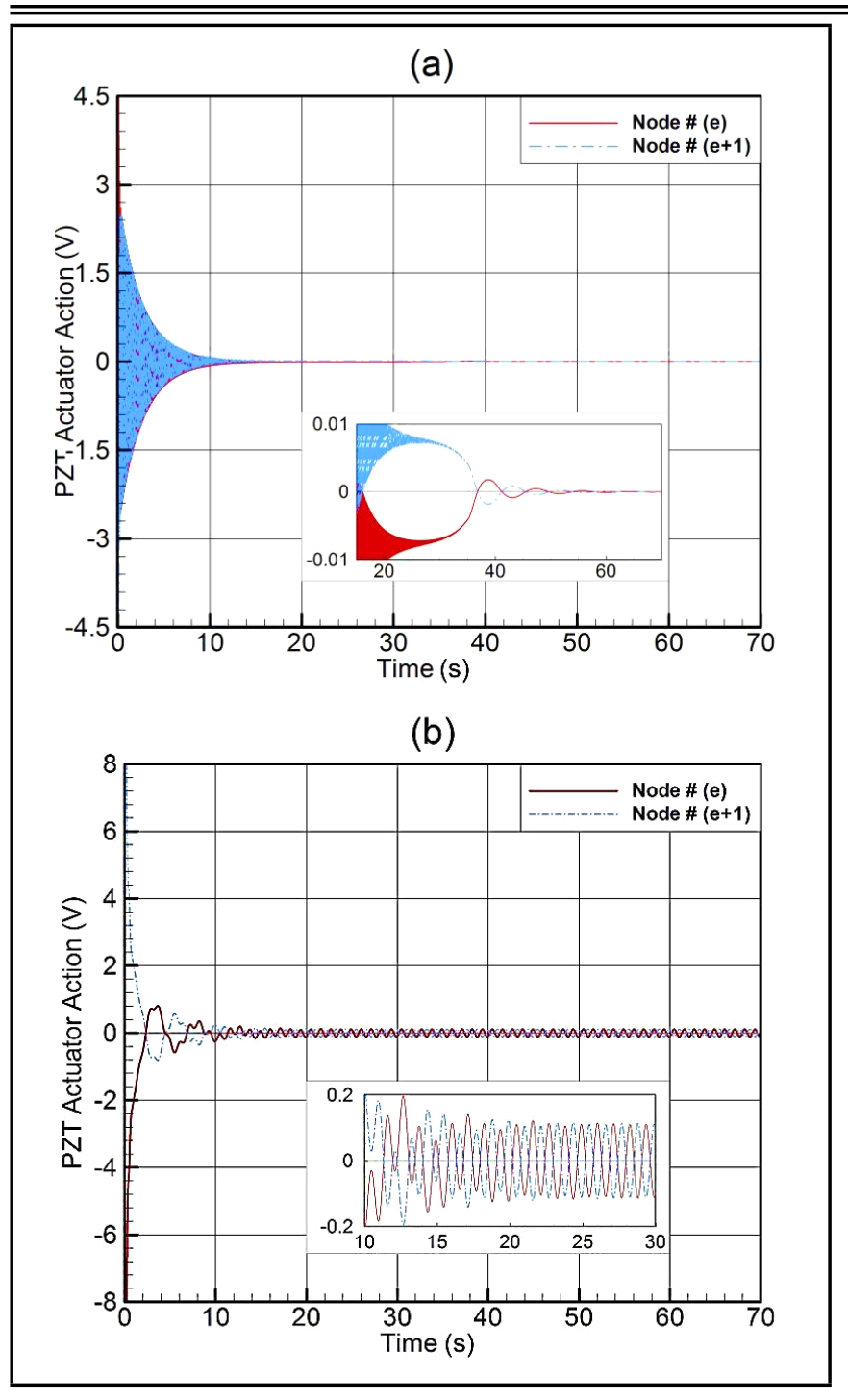

Figure 6. Time history of PZT actuation voltage; (a) Modified SMC/HigherOrder SPM and (b) SMC/CPT.

Composite Structures, $\mathbf{8 4}$ (3), 282-292, (2008). https://dx.doi.org/10.1016/j.compstruct.2007.08.007

${ }^{4} \mathrm{Li}$, Z. and Crocker M. J. A review on vibration damping in sandwich composite structures, International Journal of Acoustics and Vibration, 10 (4), 159-169, (2005). https://dx.doi.org/10.20855/ijav.2005.10.4184

5 Damanpack, A. and Khalili, S. High-order free vibration analysis of sandwich beams with a flexible core using dynamic stiffness method, Composite Structures, 94 (5), 1503-1514, (2012). https://dx.doi.org/10.1016/j.compstruct.2011.08.023

${ }^{6}$ Khalili, S., Kheirikhah, M., and Fard, K. M. Buckling analysis of composite sandwich plates with flexible core using improved high-order theory, Mechanics of Advanced Materials and Structures, 22 (4), 233-247, (2015). https://dx.doi.org/10.1080/15376494.2012.736051

${ }^{7}$ Shahravi, M., Kabganian, M., and Alasty, A. Adaptive robust attitude control of a flexible spacecraft, International Journal of Robust and Nonlinear Control, 16 (6), 287-302, (2006). https://dx.doi.org/10.1002/rnc.1051
${ }^{8} \mathrm{Hu}$, Q. Robust adaptive sliding mode attitude control and vibration damping of flexible spacecraft subject to unknown disturbance and uncertainty, Transactions of the Institute of Measurement and Control, 34 (4), 436-447, (2012). https://dx.doi.org/10.1177/0142331210394033

$9 \mathrm{Hu}, \mathrm{Q}$. Variable structure maneuvering control with time-varying sliding surface and active vibration damping of flexible spacecraft with input saturation, Acta Astronautica, 64 (11), 1085-1108, (2009). https://dx.doi.org/10.1016/j.actaastro.2009.01.009

10 Song, G. and Kotejoshyer, B. Vibration reduction of flexible structures during slew operations, International Journal of Acoustics and Vibration, 7 (2), 105-109, (2002). https://dx.doi.org/10.20855/ijav.2002.7.2107

11 Song, G. and Sethi V. Comparative study of active control of a large composite I-beam, International Journal of Acoustics and Vibration, 8 (4), 231-238, (2003). https://dx.doi.org/10.20855/ijav.2003.8.4149

12 Horodinca, M., Seghedin, N.-E., Carata, E., Boca, M., Filipoaia, C., and Chitariu, D. Dynamic characterization of a Piezoelectric actuated cantilever beam using energetic parameters, Mechanics of Advanced Materials and Structures, 21 (2), 154-164, (2014). https://dx.doi.org/10.1080/15376494.2012.680668

$13 \mathrm{Hu}, \mathrm{Q}$. Sliding mode attitude control with L2-gain performance and vibration reduction of flexible spacecraft with actuator dynamics, Acta Astronautica, 67 (5), 572-583, (2010). https://dx.doi.org/10.1016/j.actaastro.2010.04.018

14 Carrera, E. and Petrolo, M. Refined one-dimensional formulations for laminated structure analysis, AIAA Journal, 50 (1), 176-189, (2012). https://dx.doi.org/10.2514/1.j051219

15 Giunta, G., Biscani, F., Belouettar, S., Ferreira, A. J. M., and Carrera, E. Free vibration analysis of composite beams via refined theories, Composites Part B: Engineering, 44 (1), 540-552, (2013). https://dx.doi.org/10.1016/j.compositesb.2012.03.005

16 Sainsbury, M. G. (Experimental and theoretical techniques for the) Vibration analysis of damped complex structures, $\mathrm{PhD}$ dissertation, Imperial College London, University of London, (1976).

17 Meeker, T. Publication and Proposed Revision of ANSI/IEEE Standard 176-1987 ANSI/IEEE Standard on Piezoelectricity, IEEE Transactions on Ultrasonics, Ferroelectrics, and Frequency Control, 43 (5), 717-772, (1996). https://dx.doi.org/10.1109/tuffc.1996.535477

18 Mirzaee, E., Eghtesad, M., and Fazelzadeh, S. Maneuver control and active vibration suppression of a two-link flexible arm using a hybrid variable structure/Lyapunov control design, Acta Astronautica, 67 (9), 1218-1232, (2010). https://dx.doi.org/10.1016/j.actaastro.2010.06.054

$19 \mathrm{Hu}, \mathrm{Q}$. and $\mathrm{Ma}, \mathrm{G}$. Variable structure control and active vibration suppression of flexible spacecraft during attitude maneuver, Aerospace Science and Technology, 9 (4), 307317, (2005). https://dx.doi.org/10.1016/j.ast.2005.02.001 